\title{
EVOLUCIÓN Y ANÁLISIS DE UNA EXPERIENCIA DE UTILIZACIÓN DE VIDEOCONFERENCIA DE SALA Y DE ESCRITORIO
}

\section{EVOLUTION AND ANALYSIS ABOUT USES OF ROOM AND DESKTOP VIDEOCONFERENCES}

\author{
Ana Aurora Vidal Martínez \\ avidal@ciad.mx \\ Dra. Beatriz Olivia Camarena Gómez \\ betica@ciad.mx
}

Centro de Investigación en Alimentación y Desarrollo, A.C.

Carretera a la Victoria km. 0.6, Hermosillo Sonora (México)

Este trabajo describe la evolución del servicio de videoconferencia que presenta un centro de investigación en México durante los últimos diez años con énfasis en la presentación genérica de las herramientas utilizadas y los cambios tecnológicos que han revolucionado el campo de las comunicaciones. Se describen los cambios a partir de las áreas en las que se han integrado las Tecnologías de Información y Comunicación, sus límites de uso y la oportunidad que representa para potenciar la cobertura de los programas de especialización y posgrado. Para la recogida de datos se utilizó el diseño longitudinal de tendencia y el Sistema Gestionnaire Libre de ParcInformatique (GLPI). Los resultados muestran el proceso de mejora y aprendizaje que ha acompañado el servicio de videoconferencia y el potencial que representa para dos áreas sustantivas de la institución: investigación y docencia.

Palabras clave: Educación a distancia, medio de aprendizaje, sistema a tiempo real, tecnología educativa.

This paper describes evolution of videoconferencing system, in a research center in Mexico during the last ten years with emphasis on generic presentation of the tools used and the technological changes that have revolutionized the communication area. We describe changes from the areas that have been integrated Information Technology and Communication, limits of use and the opportunity presented to enhance the coverage of specialization and postgraduate programs. The longitudinal design trend System Gestionnaire Free Parc Informatique (GLPI) was used for data collection. The results show the improvement process and learning that has accompanied the videoconferencing and the potential it represents for two substantive areas of the institution: research and teaching.

Keywords: Distance learning, learning environment, real time system, educational technology.

Píxel-Bit. Revista de Medios y Educación. No 47 Julio 2015. ISSN: 1133-8482. e-ISSN: 2171-7966. doi: http://dx.doi.org/10.12795/pixelbit.2015.i47.04 


\section{Justificación.}

Hoy en día, el uso de las Tecnologías de la Información y Comunicación (TIC), se ha vuelto una característica competitiva fundamental de las organizaciones. Las instituciones educativas no son la excepción. El entorno actual les demanda estar equipadas y actualizadas en TIC para que las tareas que realizan y los servicios que ofrecen (a nivel organizativo y educativo) sean de la mejor calidad y eficiencia (Carmena \& Navarro, 2013).

El servicio de videoconferencia (SV), como parte importante de las TIC, se define como «el conjunto de hardware y software que permite la conexión simultánea en tiempo real por medio de imagen y sonido que hacen relacionarse e intercambiar información de forma interactiva a personas que se encuentran geográficamente distantes, como si estuvieran en un mismo lugar de reunión» (Cabero, 2000, p. 98). Por tanto, su propósito es facilitar la intercomunicación del personal y clientes de la organización que están ubicados en lugares geográficos distantes.

Generalmente, las actividades y servicios que ofrecen los centros de investigación e instituciones de educación superior se agrupan en investigación, docencia, vinculación y administración. Para facilitar la interacción del personal que se desempeña en cada una de estas cuatro áreas, sobre todo cuando están ubicados en lugares geográficos distantes, se invierte en equipos tecnológicos y en programas específicos para la transmisión y recepción de audio, video y datos que requiere el SV. El requisito para que se realice la comunicación a distancia es que todos los sitios cuenten con acceso a la señal de Internet y con el equipo adecuado para realizar la conexión en tiempo y forma.
Además, los usuarios o personal interesado deben conocer las características mínimas de las tecnologías integradas al SV.

Este documento describe la evolución del servicio de videoconferencia en un centro de investigación en México: la presentación genérica de las herramientas existentes, la evolución del servicio en cuanto a la tecnología involucrada que ha revolucionado el campo de las comunicaciones (medios físicos, software y esquemas de uso de tales recursos), las características de los participantes o usuarios de tal servicio y su entorno así como los instrumentos utilizados para la recopilación de la información. Se documentan los cambios que presenta el SV durante los últimos diez años a partir de las áreas en las que se ha integrado, sus límites de uso y la oportunidad que representa para potenciar la cobertura de ciertas actividades sustantivas del centro. Para la recogida de datos se utilizó el diseño longitudinal de tendencia y el Sistema Gestionnaire Libre de ParcInformatique (GLPI) (Indepnet Association Use GLPI, 2013) como soporte para el análisis de tendencia. La información corresponde al número de sesiones por área; el tipo de equipo y videoconferencia utilizada, el personal participante en la misma y la percepción que los usuarios y el responsable del servicio tienen al respecto. Se observan y analizan las variables a través del tiempo en una población determinada en la cual los individuos pueden cambiar pero la población sigue siendo la misma (Albert, 2007). Se trata de un estudio de tipo no experimental en el cual se observan y analizan los fenómenos tal y como se presentan en el contexto natural, proceso de investigación sustentado «en una búsqueda empírica y sistemática de información cuando las manifestaciones del fenómeno ya han ocurrido y por tanto las 
variables son inherentemente no manipulabes» (Kerlinger \& Lee Howard, 2002, p. 504).

\section{Potencial de la videoconferencia de sala y de escritorio.}

En las áreas de docencia, investigación y vinculación se propician diversos tipos de redes con objetivos variados y donde está presente personal con formaciones disciplinarias distintas. En estos vínculos donde interactúan varias personas puede darse la colaboración y la generación de nuevos conocimientos de manera que las instituciones y los involucrados se vean favorecidos por la generación del valor agregado a través de la unión de esfuerzos y experiencias.

Sin duda, una forma de impulsar los procesos de innovación en la educación superior vinculando la tecnología, la pedagogía y la organización es haciendo posible las diversas opciones de trabajo colaborativo que permite la formación en línea (Hernández, González \& Muñoz, 2014). Hoy en día, por ejemplo, se sabe que la educación de imagen propiciada por los medios masivos y la alfabetización audiovisual como complemento de la alfabetización tradicional, son experiencias de aprendizaje importantes de analizar (Castillo \& Cabrerizo, 2006).

En ese sentido es importante analizar el potencial que representa el SV de sala y de los sistemas de escritorio, lo cual ha sido planteado desde hace ya más de quince años como un buen complemento y recurso didáctico que ayuda al alumno en su aprendizaje y que tiene connotaciones afectivas (Sevillano, Carpio \& Sánchez Arroyo, 1998). No obstante, hay quien considera que este medio por sí mismo no es un instrumento de aprendizaje. Lo que convierte en educativo a un medio es justamente la planificación y el diseño didáctico de los contenidos que permiten que se cumpla la función educativa, lo que implica considerar aspectos metodológicos, instrumentales y técnicos (Chacón, 2003). Posteriormente, Llano, Ainciburu y Lázaro(2011) indican que es necesario considerar esta herramienta como lo que es, un medio de difusión de los contenidos de aprendizaje. E igual, Martín, López y García (2012) advierten de la necesidad de formar a los tutores en metodologías para el uso de esta tecnología, con el fin de que puedan aprovechar toda la capacidad pedagógica del recurso.

En estudios más recientes, se concluye que es posible obtener resultados positivos en el proceso de enseñanza-aprendizaje al utilizar videoconferencia considerando todos los factores que intervienen en el proceso de comunicación educativa (Álvarez, Ochoa, Salado \& Soto Bernal, 2013). También en casos y situaciones específicas se reconocen las ventajas del SV. Por ejemplo, Duro (2013) reconoce que la videoconferencia permite conectar a los alumnos en situación de aislamiento por prescripción médica a un contexto educativo cotidiano y Córdova, Staff, Cubillas y Stegaru (2013) destacan que es un medio y/o método útil en la enseñanza de medicina en las asignaturas preclínicas. También en sistemas escolarizados de mayor cobertura, como es el nivel básico, la videoconferencia es cada vez más utilizada, como reconocen Sáez y Ruiz (2014) al señalar que esta tecnología se utiliza cada vez más en el aprendizaje de medio natural, social y cultural en una escuela primaria. 
Con tales antecedentes, se revisan los cambios que presenta el SV en el centro de interés con el fin de considerar su evolución y particular incidencia en el área educativa

\section{Antecedentes de servicio de videoconferencia en institución.}

En México, los 27 Centros que constituyen el Consejo Nacional de Ciencia y Tecnología (CONACYT) inauguran de manera oficial la red de videoconferencia el año 2003, no obstante, hubo experiencias de conexión previas, como fue el caso del centro considerado en este trabajo. A la fecha, la mayoría de los centros de investigación y educación superior del país han incorporado nuevas tecnologías como parte de un proceso de renovación continua que pretende mejorar la calidad de la emisión e intercomunicación a distancia.

Al empezar a ofrecerse el SV la red enlazaba a los 27 Centros y oficinas centrales del Consejo Nacional de Ciencia y Tecnología (CONACYT) y en la segunda fase empiezan a sumarse las oficinas regionales y subsedes de este sistema. Sin embargo, no se tiene acceso a información que proporcione la cantidad y datos del equipo tecnológico disponible por cada centro en esos primeros años, sólo a la del centro de interés.

Dicho centro empieza las actividades de interconexión con un equipo multisitio ubicado en la unidad sede que permitía la interacción simultánea con máximo tres sitios. Ese primer equipo tenía como característica principal la movilidad, es decir, se podía transportar de un lugar a otro para realizar la sesión en el área que solicitaba el servicio. En el transcurso del año 2008 la institución transita a una segunda etapa al adquirir cinco equipos -uno multisitio y cuatro de conexión punto a punto- que fueron instalados en algunas de sus subsedes en base a tres criterios: la capacidad de los enlaces utilizados (ancho de banda); la velocidad de comunicación (desde $384 \mathrm{KBps}$ hasta los 2Mbps); y el número de empleados por unidad dado el propósito de facilitar el servicio a un mayor número de usuarios. Se mantuvo la opción multisitio en la unidad sede y se instalaron los equipos de conexión punto a punto. A partir de entonces, la unidad central donde quedó el equipo multisitio brinda la posibilidad de realizar reuniones en tiempo real con el personal de las subsedes a conveniencia, es decir, puede realizarse la interlocución simultánea con una o más subsedes.

La nueva tecnología instalada en esta segunda fase representó un ahorro importante en tiempo y en recursos financieros a la institución. En relación al tiempo, la reunión se agenda y se realiza de manera casi inmediata estando todos enterados y de acuerdo; en cuanto a recursos financieros, se redujeron los gastos de transporte, hospedaje y alimentación en los que incurría la institución cuando se realizan reuniones cara a cara con el personal de las sub-sedes. También, con esta inversión las unidades subsedes tienen la posibilidad de realizar conexiones punto a punto con cualquier parte del mundo (esto es con un solo sitio a la vez).

El año 2012 se transita a una tercera etapa del SV que prevalece hasta el día de hoy. La nueva inversión responde al crecimiento presentado por investigación y docencia, dos de las áreas sustantivas del centro que también inciden en las actividades que realizan las otras dos áreas importantes del centro, vinculación y administración. La matrícula escolar y el número de personal 
contratado en investigación y administración se eleva: de 86 estudiantes en el 2008 se pasa a 135 en el 2012; y de 378 empleados en el 2008 a 458 en el 2012. El servicio es demandado por un mayor número de personas en apoyo a más actividades. La cantidad de solicitudes del servicio se eleva de manera significativa al grado de rebasar la posibilidad de atención con el equipo disponible hasta entonces en la unidad sede. Se refuerza entonces, en la unidad central con tres equipos multisitio que fueron instalados en el aula de docencia; sala de usos múltiples y auditorio central (Imagen 1) e incorporando el servicio de videoconferencia de escritorio.

La asignación de cada sala a quien la solicita, está en función del número de participantes en la videoconferencia. La primera sala tiene capacidad para 24 personas como máximo; la segunda permite hasta 80 ; y la tercera 220 participantes. Todas ellas están acondicionadas para alojar de manera cómoda a los participantes/usuarios del servicio así como el equipo de control de audio $\mathrm{y}$ video, que es el que permite capturar y controlar las imágenes y los sonidos que habrán de transmitirse hacia el o los sitios remotos. El ambiente se asemeja a una sala normal para reuniones o conferencias, su comodidad depende de la calidad de la instalación, para lo cual se ha cuidado que los asistentes no se intimiden por la tecnología presente en la sala.

Actualmente, el centro cuenta con el servicio de videoconferencia de sala y de escritorio. Hay nueve equipos en total, distribuidos en la sede principal y cuatro subsedes (Tabla 1). En la sede principal (Hermosillo), hay cuatro equipos multisitio y uno de conexión punto a punto. Las subsedes de Guaymas, Mazatlán, Culiacán y Cuauhtémoc, cuentan con un equipo punto a punto.

En relación a una sesión de videoconferencia de escritorio -aquél que permite la transmisión de audio, vídeo y datos a través de una computadora personal (Salinas \& De Benito, 2004), es importante precisar que el usuario requiere de una computadora con acceso a Internet, micrófono, bocinas, cámara web y descargar un pequeño programa de fácil instalación en su equipo.

Los sistemas que conformaron el nuevo servicio en el centro de referencia, fueron seleccionados en base a los reportes y recomendaciones de empresas independientes (Business-Software, 2012; WebConferencing, 2011; Urbina et al., 2010). Para orientar el proceso de selección hacia el sistema más óptimo se tomaron en cuenta las características de cada sistema, por ejemplo:
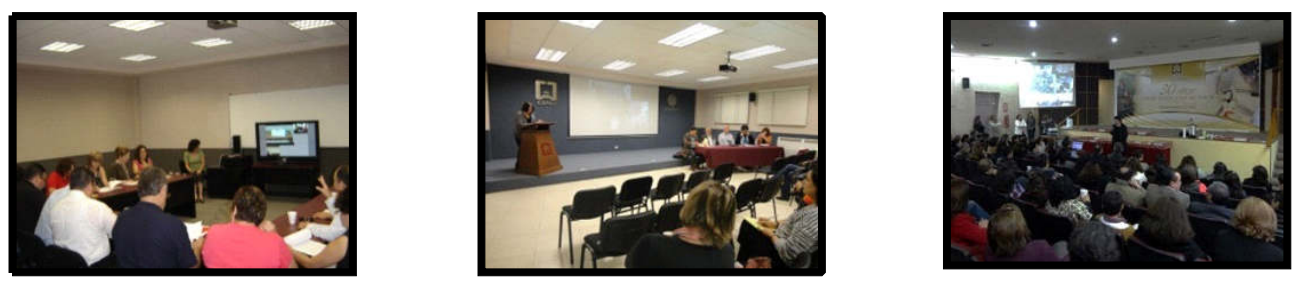

Imagen 1. Salas fijas de videoconferencia en sede principal. 


\begin{tabular}{|c|c|c|c|}
\hline UNIDADES & 2003 & 2008 & 2012 \\
\hline \multirow{2}{*}{ Hermosillo } & EquipoVCmultisiti & EquipoVCmultisiti & EquipoVCmultisitio (2) \\
\hline & $o(1)$ & o (1) & Equipo de VC (1) \\
\hline Mazatlán & & Equipo VC (1) & \\
\hline Guaymas & & Equipo VC (1) & \\
\hline Cuauhtémoc & & Equipo VC (1) & \\
\hline Culiacán & & Equipo VC (1) & \\
\hline
\end{tabular}

Tabla 1.Red de equipos de videoconferencia instalados del 2003 al 2012 en cada unidad.
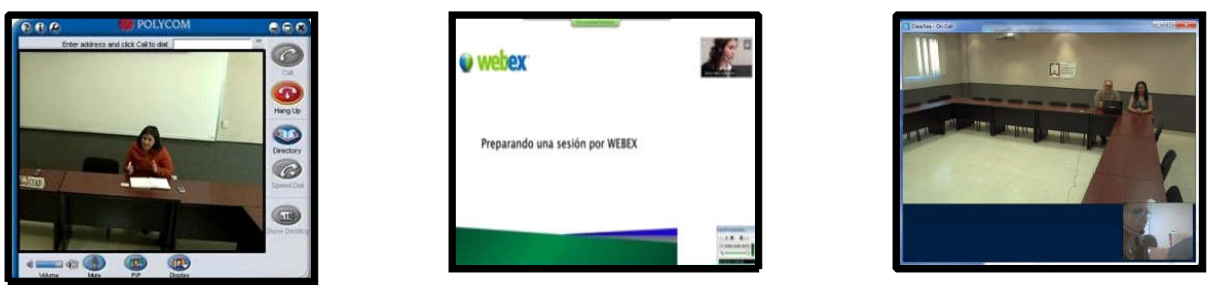

Imagen 2. Videoconferencia de escritorio.

la seguridad de la información, calidad de audio y video, ayuda y soporte, facilidad de uso, número máximo de participantes, soporte remoto, manuales en línea, uso compartido de aplicaciones y herramientas, compatibilidad, configuración ajustable y precio, (Watson, 2001). PVXPolycom (Imagen 2, primer recuadro). Proporciona una tecnología de videoconferencia para PC que permite transmitir video y audio bidireccionalmente, con la limitante de que no es compatible con todos los equipos de videoconferencia (PVX 2005).

Sistema WEBEX CISCO (Imagen 2, segundo recuadro). Es una herramienta de colaboración a través de Internet que permite realizar vídeo conferencias de escritorio desde cualquier computadora o dispositivos móviles, dejando enviar y recibir audio, video y datos, grabar la sesión, compartir archivos, modificarlos en línea, compartir aplicaciones diversas con un grupo de herramientas de administración de la reunión que facilitan la interacción y el orden durante la sesión (Webex. CISCO 2011). El inconveniente aquí es que no es compatible con los equipos de videoconferencia. El año 2009, el centro adquiere una licencia del sistema WEBEX con capacidad de hasta 25 usuarios.

Sistemas de escritorio Clear Sea (2012). LifeSize Logitech(Imagen 2, tercer recuadro). Esta es una herramienta totalmente 


\begin{tabular}{|c|c|c|c|c|}
\hline UNIDADES & 2004 & 2009 & 2010 & 2014 \\
\hline Hermosillo & PVX de Polycom & Webex de Cisco & & Clear Sea de LifeSize \\
\hline Mazatlán & \multicolumn{3}{|r|}{ PVX de Polycom } & \\
\hline Guaymas & \multicolumn{3}{|c|}{ PVX de Polycom } & \\
\hline \multicolumn{5}{|l|}{ Cuauhtémoc } \\
\hline \multicolumn{5}{|l|}{ Culiacán } \\
\hline Delicias & & PVX de Polycom & & \\
\hline
\end{tabular}

Tabla 2. Red de sistemas de videoconferencia de escritorio instaladas por unidad, 2004-2014.

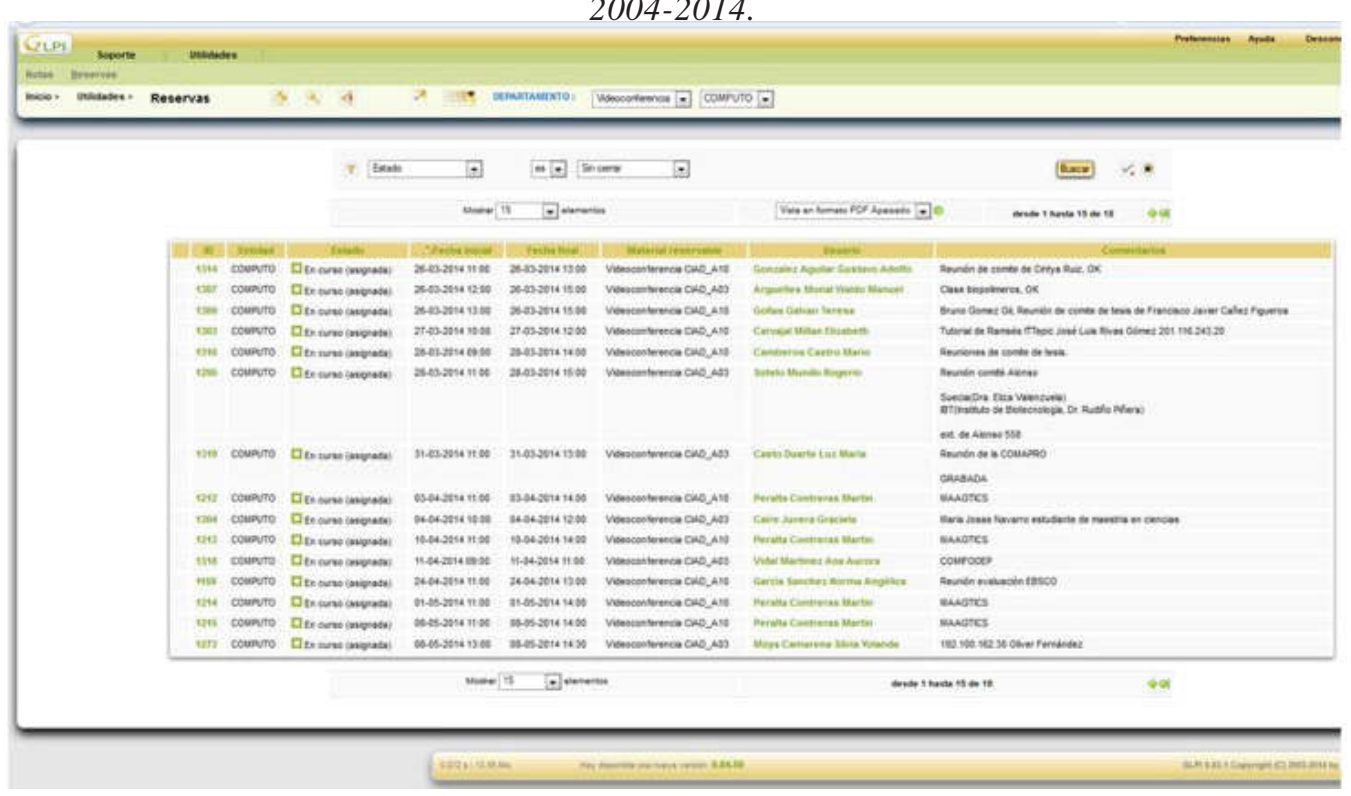

Imagen 3. Sistema para solicitar el servicio de videoconferencia.

transversal que permite la comunicación entre todas las marcas de equipos de videoconferencia, la comunicación es vía software como un punto más en las reuniones, como si se tratara de un equipo de videoconferencia, el cual es instalable en todos los dispositivos existentes (Clear Sea 2012. LifeSize Logitech).
En resumen la sede y subsedes del centro cuentan con los siguientes sistemas de videoconferencia de escritorio (Tabla 2): en la unidad central (sede Hermosillo) Webex de Cisco, PVX de Polycom y Clear Sea de LifeSize; en las sub-sedes, se cuenta con licencia PVX de Polycom (Mazatlán, Guaymas y Delicias).

Píxel-Bit. Revista de Medios y Educación. No 47 Julio 2015. ISSN: 1133-8482. e-ISSN: 2171-7966. doi: http://dx.doi.org/10.12795/pixelbit.2015.i47.04 
Todo parece indicar que el cambio con fines de mejora que presentan los recursos a lo largo de los años responde a la observación, a la experiencia práctica y las investigaciones realizadas.

\section{Lineamientos base para monitoreo del} Servicio de Videoconferencia.

El GLPI corresponde a una aplicación Web -solución de software abierto- que ataca los principales problemas de gestión del inventario informático (administración de recursos de hardware, software, usuarios, suministros e incidencias); y facilita el registro de las solicitudes así como llevar a cabo la gestión del inventario informático y de soporte técnico, como se aprecia en la pantalla de inicio del mismo (Imagen 3).

En la Imagen 3, se observa cómo en función de la variable de interés, se definen las clasificaciones que, en el caso que nos ocupa, son tres:

En función del tipo de videoconferencia, hay dos modalidades:

Videoconferencia de sala. Se utiliza un equipo de videoconferencia

- Videoconferencia de escritorio. Se utiliza un software y computadora

En función del número de sitios enlazados, dos modalidades más:

Punto a punto. El enlace se realiza entre dos sitios

Multipunto. Cuando son más de dos los sitios enlazados. En el caso de la videoconferencia de sala es necesario un equipo que sea capaz de enlazar a todos los sitios que participen y para el tipo de escritorio se requiere de una licencia de software con el número de usuario que vayan a participar en la sesión.
En función del tipo de participación, dos posibilidades:

Unidireccional. Situación en la que hay un emisor y varios receptores y la comunicación fluye solo en un sentido.

Multidireccional. Situación donde todos los participantes son emisores y receptores.

\section{Descripción y análisis de seguimiento del servicio de videoconferencia.}

\section{Tendencia del Servicio de} Videoconferencia en general y por área.

En la Gráfica 1 se plasma el número de videoconferencias realizadas por cada una de las áreas sustantivas del centro del 2004 al 2013. En primer lugar, llama la atención el aumento constante de sesiones en relación al total de sesiones del año previo tanto a nivel global como en relación a cada área, lo cual se vincula con el comportamiento creciente de la matrícula escolar y la contratación de más personal (administrativo y de investigación). De manera natural, el mayor número de personal generó o provocó también una mayor demanda del servicio.

En los primeros cinco años (2004-2008), las áreas que realizaron el mayor número de sesiones fueron las áreas de administración y la de investigación; y los últimos cinco años (2009-2013), fueron docencia e investigación.

Tendencia de servicio por Tipo de Videoconferencia (sala o escritorio).

Los datos de la Gráfica 2 muestran que se realizaron más sesiones tipo sala que escritorio, lo cual se liga con las características de cada tipo de videoconferencia y no tanto por la calidad o potencialidaddel servicio. Es 


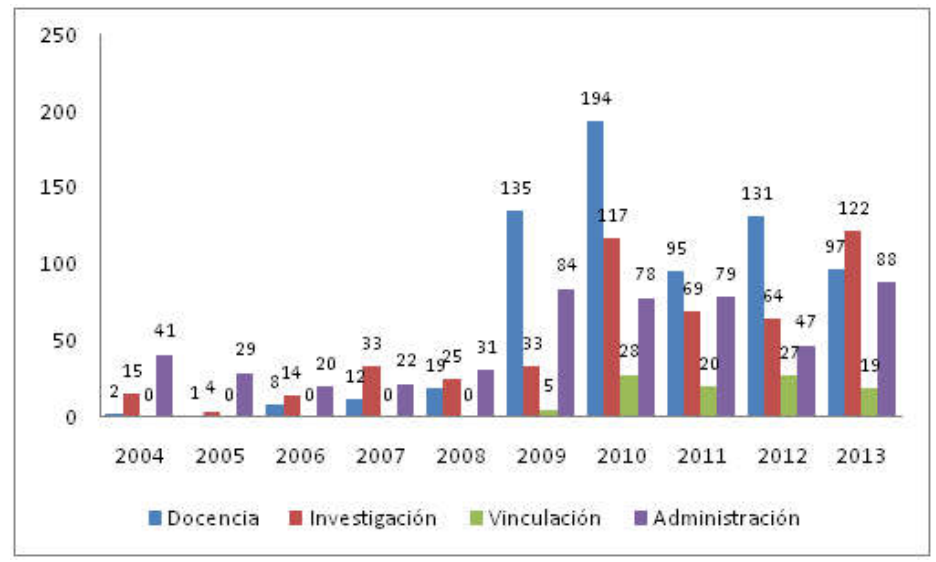

Grafica 1. Videoconferencia de sala y escritorio por área.

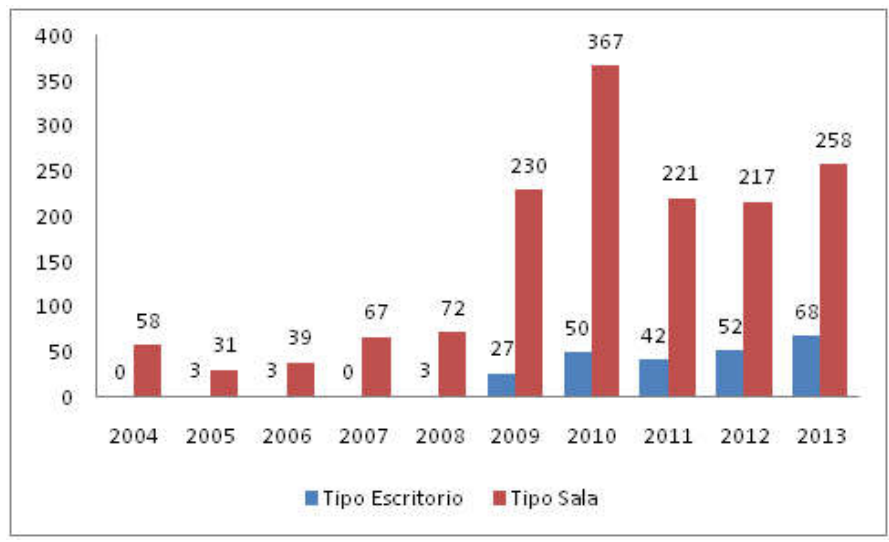

Grafica 2. Tipos de Videoconferencia.

decir, la demanda se inclina más por las necesidades del usuario así como por la organización y la estrategia considerada por éste para llevar a cabo cada una de las sesiones.

\section{Tendencia del servicio de videoconferencia} por número de sitios enlazados.

Como se puede apreciar en la Gráfica 3, el número de videoconferencias realizadas punto a punto es superior a las sesiones realizadas con el uso del equipo multipunto. Este dato, permite concluir que este servicio se utiliza en mayor medida para hacer conexiones con un solo sitio en cada sesión. Lo cual también es lógico ante el mayor número de personal y el equipo instalado que les permite realizar tal interacción en base a sus particulares necesidades académicas y/ o de investigación. 


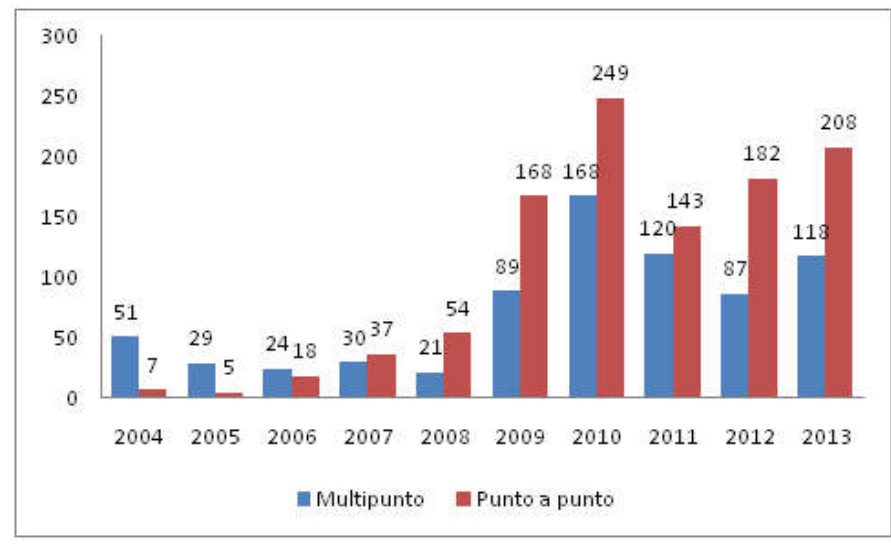

Grafica 3. Videoconferencias en función del número de sitios enlazados por sesión.

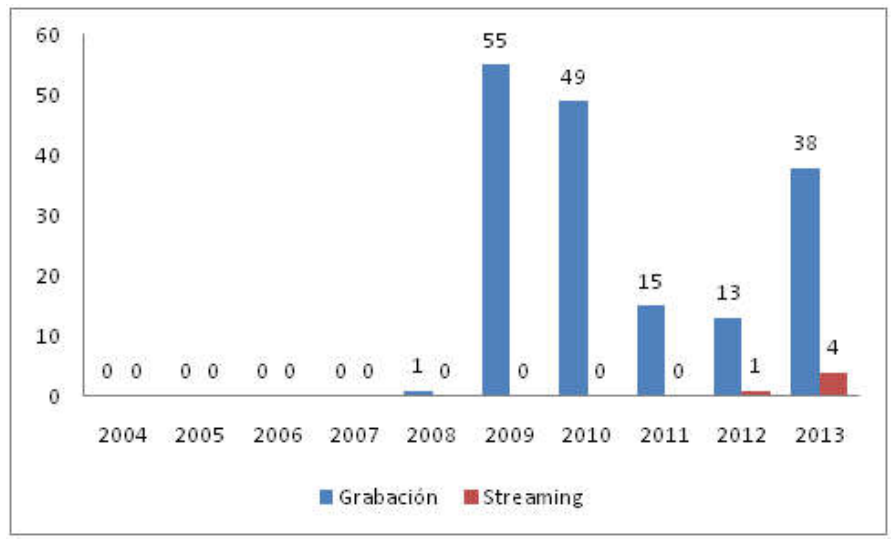

Grafica 4. Recursos auxiliares solicitados para videoconferencia.

Tendencia de uso de recursos auxiliares del Servicio de Videoconferencia.

En la Gráfica 4 la información representa al uso de recursos auxiliares solicitados para una sesión. El usuario puede solicitar al responsable de este servicio que su sesión sea grabada o transmitida por Internet.

La frecuencia de uso de los recursos auxiliares, sobre el número de sesiones pasa del $21 \%$ en el 2009 al $12.8 \%$ en el 2013. Al parecer, las características y propósitos de cada sesión, tiene que ver con los recursos auxiliares solicitados. Por ejemplo, el contenido de las sesiones con fines educativos, aumenta el interés de grabarla para aprovechar el potencial de reproducción que permite esta tecnología obviamente una vez autorizados los permisos institucionales y de autoría correspondientes grabación que 
se puede utilizaren procesos de enseñanzaaprendizaje posteriores. Al grabarse una sesión de este tipo se puede reproducir posteriormente a conveniencia para complementar, por ejemplo, la labor tutorial o al ser transmitida por streaming los estudiantes solo requieren de una computadora con Internet para recibir la señal de la clase (audio, video y datos). Esta oportunidad que representa el servicio de videoconferencias y auxiliares, tiene gran potencial para el área de docencia.

\section{Conclusiones.}

Tras una experiencia de casi once años donde el SV ha evolucionado en el caso en observación. El servicio es muy conocido por los empleados del centro y lo solicitan de manera cotidiana $y$ ante cualquier eventualidad, lo cual se asocia con una valoración positiva del funcionamiento del servicio. El SV ha evolucionado de manera natural en respuesta a las necesidades de intercomunicación del personal que están vinculadas principalmente con actividades de administración, planeación y control administrativo. Sin embargo, falta ahora mejorar la calidad y cobertura del servicio sobre todo para planear su crecimiento de manera anticipada en vistas a reforzar el quehacer de las dos áreas sustantivas del centro correspondientes a docencia e investigación. En ese tenor, será necesario incorporar tareas y acciones para reforzar, diversificar y/o complementar la interacción en redes de investigación y para lograr concretar la oferta de programas de especialización y posgrado en la modalidad a distancia.

Sin duda, los cambios que presenta el SV en el centro de interés muestran una evolución importante y una aceptación positiva por parte del personal. Es necesario dar el siguiente paso al observar las amplias posibilidades que tiene hoy en día este sistema para el área educativa. Habrá que diseñar y hacer realidad los programas de especialidad y/o posgrado en línea, proyecto hoy por hoy viable pero que sigue quedando como una tarea pendiente. El desafío está en definir con claridad y precisión el cómo se puede transitar de lo posible al hecho para entonces sí, una vez en marcha en el campo de la enseñanza, valorar el impacto de la modalidad a distancia en los programas de posgrado.

\section{Referencias bibliográficas.}

Albert, M.J. (2007). La investigación Educativa: Claves Teóricas. España: McGraw-Hill/Interamericana de España, S.A. Álvarez, E.P., Ochoa, R.I., Salado, L.I.\& Soto Bernal, R.A. (2013). La interacción de factores del modelo de videoconferencia y su influencia en el proceso de enseñanzaaprendizaje. Revista Internacional Administración \& Finanzas, 6 (4). Recuperado de http://papers.ssrn.com/sol3/ papers.cfm?abstract_id=2157171

Business-Software (2012). Top $10 \mathrm{Web}$ Conferencing Software Vendors Revealed. Recuperado de http://www.networkingplanet.com/Uploads/documents/5775acc63601-48d2-9411-355a98f4d5da.pdf

Cabero, J. (2000). Nuevas Tecnologías aplicadas a la educación. Madrid: Síntesis. Carmena E. \&Navarro, J. (2013). Evaluación del uso de la tecnologías en la enseñanza universitaria a distancia de la UNED.Revista Iberoamericana de educación a distancia, 16 (2), 73-106.doi: http://dx.doi.org/10.5944/ ried.16.2.9907 
Castillo, S. \& Cabrerizo, J. (2006). Formación del profesorado en Educación Superior: Desarrollo Curricular y Evaluación (Vol. II). Madrid, España: McGraw Hill.

Chacón, A. (2003). La videoconferencia: conceptualización, elementos y uso educativo.RevistaEtic@net publicación en línea, Año I (2). Recuperado de http:// www.ugr.es/ sevimeco/revistaeticanet/ $\mathrm{Num} \mathrm{ero} 2 / \mathrm{Art}$ i c u 1 o s / L a \% 20 videoconferencia.pdf

Clear Sea (2012).Life Size Logitech.Administrator Guide. Recuperado de http://www.lifesize.com/ /media/ Documents/Product\%20Documentation/ ClearSea/Guides\%20and\%20Reference/ Clearsea\%20Server\%20Administrator $\% 20$ Guide $\% 20 \mathrm{EN}$.ashx

Córdova,A., Staff, C., Cubillas, F. \& Stegaru, M. (2013). Uso y utilidad de la videoconferencia en la enseñanza de asignaturas preclínicas de medicina en la Universidad Latina de Panamá (ULAT), Elsevier México.Investigación en Educación Médica,2 (1), 7-11. Recuperado de http:// riem.facmed.unam.mx/sites/all/archivos/ V2Num01/03_AO_CORDOVA.PDF

Duro, D.C. (2013). La videoconferencia en el contexto de las aulas hospitalarias de la CARM. Trabajo Máster Universitario en Psicología de la Educación. Murcia: Universidad de Murcia. Recuperado dehttp:/ /digitum.um.es/xmlui/bitstream/10201/34960/ 1/La\%20Videoconferencia\%20en\%20el\%2 0 contexto $\% 2$ 0de $\% 201$ as $\% 20$ Aulas $\%$ $20 \mathrm{Hos}$ i i a larias $\% 20 \mathrm{de} \% 201$ a $\%$ 20CARM\%20Diana\%20Clara\%20Duro\%2011.pdf

Hernández, N., González, M. \& Muñoz, P.C. (2014). La planificación del aprendizaje colaborativo en entornos virtuales.
Comunicar, 42, 25-33. Recuperado de http:// w w w.revis t a com un i a r c o m/ index.php?contenido $=$ detalles \&numero $=42$ \&articulo=42-2014-02 $\gg$ articulo=42-2014-02 . doi: http://dx.doi.org/10.3916/C42-2014-02

Indepnet Association Use GLPI (2013). Version 0.84. Publicado por Creative Commons. Recuperado de http://www.glpiproject.org/

Kerlinger, F. \& Lee Howard, B. (2002): Investigación del comportamiento. Métodos de investigación en ciencias sociales. México: McGraw-Hill.

Llano, J., Ainciburu, M.C. \& Lázaro, O. (2011). La Enseñanza de Español a través de Videoconferencias de Escritorio, Integración en las Diferentes Modalidades de Aprendizaje y Desarrollo de Competencias. Cuadernos Comillas, 2, 51-68.

Martín, A.M., López, M.A. \& García, A. (2012). Red de Innovación: La videoconferencia como recurso en el apoyo tutorial y en el aprendizaje autónomo.Revista Iberoamericana de Tecnologías del Aprendizaje,7(2), 95-101. Recuperado de: h t t p : / / ri t a.det.u vigo.e s / i n d e x.ph p ? c o n t e n t $=\mathrm{Nu} \mathrm{m}$ Pub\&idiom $=$ Es \&visualiza $=1 \&$ volumen $=$ 7\&numero=2\&orden $=$ desc $\gg$ orden $=$ desc

PVX (2005). Internet and Intranet Colling with Polycom PVX 8.0.1.Polycom. Edition: 3725-23299-001/A. Recuperadode http:// supportdocs.polycom.com/PolycomService/ support/global/ documents/support/user/ products/video/pvx_internet intranet_calling.pdf

Sáez, J.M. \& Ruiz, J.R. (2014). La enseñanza de las ciencias naturales y sociales a través de la videoconferencia interactiva. Estudio de caso en educación primaria. Pixel-Bit. Revista de medios y educación, 44, 35-49. Recuperado de http://acdc.sav.us.es/pixelbit/ 
images/stories/p44/completo.pdf. doi: http:/ /dx.doi.org/10.12795/pixelbit.2014.i44.03

Salinas, J. \& De Benito, B. (2004). Diseño de acciones formativas soportadas en videoconferencia. En J. Salinas, J. Cabero \& J.I. Aguaded (Eds.). Tecnologías para la educación: diseño, producción y evaluación de medios para la formación docente (pp. 231-248). Madrid, España: Alianza Editorial.

Sevillano, M.L., Carpio, J. \&Sánchez, E. (1998). La Videoconferencia en la Universidad Nacional de Educación a Distancia: Análisis y Resultados. Revista Iberoamericana de Educación a Distancia,1 (2), 71-99.

Urbina, S., Arrabal, M., Martín, A., Ordinas, C., Pons, S. \& Rodríguez, S. (2010). Análisis de herramientas de videoconferencia de escritorio para la docencia en pequeño grupo y la tutoría en entornos de educación superior.XIII Congreso Internacional Edutec 2010: E-Learning 2.0: Enseñar y Aprender en la Sociedad del Conocimiento. Bilbao, España. Recuperado de http:/gte.uib.es/pape/gte/ sites/gte.uib.es.pape.gte/files/ ANALISIS\%20DE\%20HERRAMIENTA S \% 20 D E \% 20 V I D E O C O N F E RENCIA\%20DE\%20ESCRITORIO\%20PARA\% 20LA\%20DOCENCIA\%20EN\%20PEQUEN\% CC $\% 83$ O \% 20 GRUPO.pdf » 20 DE $\%$ 20VIDEOCONFERENCIA\%20DE\%20ESCRI TORIO \% 20 PAR A \% 20 L A \% 20 D O C E N C I A \% 20 E N \% 20 P E Q U EN $\%$ CC\%830\%20GRUPO.pdf

Watson, B.C. (2001). The Distance Learning Project. Journal of Library Administration, 34(3-4), 325-328. doi: 10.1300/J111v34n03_12

Webex, CISCO. (2011). CISCO Webex Administración del Sitio: Guía del usuario. In CISCO (Ed.): CISCO.

Web-Conferencing (2011). Web Conferencing Buyer's Guide - Par t 1 Must have Features of Web Conferencing
Software. Recuperado de http:// webconferencing.org/offer/webconferencing-buyers-guide-part-1-musthave-features-of-web-conferencingsoftware/

Fecha de recepción: 25-02-2015 Fecha de evaluación: 28-03-2015 Fecha de aceptación: 28-04-2015 